\title{
Gains in native species promote biotic homogenization over four decades in a human-dominated landscape
}

\author{
Jenny L. McCune ${ }^{1 *}$ and Mark Vellend ${ }^{2}$ \\ ${ }^{1}$ Department of Botany and Biodiversity Research Centre, University of British Columbia, 3529-6270 University Blvd, \\ Vancouver BC V6T 1Z4, Canada; and ${ }^{2}$ Département de biologie, Université de Sherbrooke, Sherbrooke QC J1K 2R1, \\ Canada
}

\begin{abstract}
Summary
1. A long-term perspective is needed to understand how disturbance is affecting plant communities in human-dominated landscapes. Increased human disturbance often results in declining local native species richness, gains in exotic species and a decline in beta diversity. However, it is far from certain whether a general decline in plant diversity is occurring across all disturbed landscapes, and knowledge gaps remain concerning how the spread of exotic species influences beta diversity over long time-scales.
\end{abstract}

2. We resurveyed 184 vegetation plots in three broad vegetation types on southern Vancouver Island, Canada, originally surveyed in the late 1960s. This landscape has experienced a high degree of human disturbance over the past 40 years due to urbanization. We examined changes in total diversity, local diversity and beta diversity over time. We also compiled information on the traits of each species and tested for correlations between traits and plant species success over four decades.

3. We found striking increases in local and total plant species richness driven by both native and exotic species. The most successful species tended to be exotic, disturbance tolerant, shade tolerant and shrubs. Biotic homogenization occurred, but not as a result of exotic species colonization, instead being significantly correlated with gains in native species. The loss in beta diversity has resulted in a shrinking of the gradient of vegetation types, blurring the distinction between them.

4. Synthesis. Our study shows that human-mediated disturbance is the dominant driver of plant community changes, but the net result has actually been an increase in richness, for each plot and for all plots pooled, and for both natives and exotics, despite a decline in variability among plant communities on the landscape. Contrary to conventional definitions of biotic homogenization, this decline in beta diversity was not correlated with the spread of exotic species, but with the colonization of common, disturbance-tolerant natives.

Key-words: beta diversity, determinants of plant community diversity and structure, exotic species, human disturbance, life-history traits, resurvey, semi-permanent plots, Vancouver Island

\section{Introduction}

Habitat loss, disturbance and the introduction of exotic species to new areas are causing declines in biodiversity on a global scale (e.g. McKinney \& Lockwood 1999; Butchart et al. 2010). Changes in diversity at the local scale are more difficult to quantify because of the scarcity of data on species presences in small-scale plots over time periods longer than a decade (Sax \& Gaines 2003). Recently, plant ecologists have been addressing this gap using so-called 'legacy' data (Vellend et al. 2013) to quantify long-term changes in plant

*Correspondence author. E-mail: jmccune@ biodiversity.ubc.ca community diversity at the local scale (e.g. Smart et al. 2006; Rogers et al. 2008; Keith et al. 2009; Damschen, Harrison \& Grace 2010). Most have found declines not only in plot-level diversity (alpha diversity) and regional diversity (gamma diversity), but also in beta diversity: that is, a reduction in variability in community composition across plots. However, these studies are still relatively few, and it is far from certain whether a general decline in plant diversity is occurring across all landscapes. Some legacy studies from Europe have found increases in local and regional diversity (Van Calster et al. 2007; Aggemyr \& Cousins 2012).

Loss in beta diversity occurs via biotic homogenization, whereby widespread generalist species colonize plots across a region, while more specialist species are lost, reducing the 
variability in community composition (McKinney \& Lockwood 1999). Loss of diversity at this level can go unnoticed because beta diversity can decline even though alpha and gamma diversity remain unchanged or even increase (Olden \& Poff 2003; Van Calster et al. 2007; Keith et al. 2009). Biotic homogenization as originally defined is '... the replacement of local biotas with non-indigenous species, usually introduced by humans' (McKinney \& Lockwood 1999). But studies using native and exotic species lists to calculate beta diversity between localities, watersheds or states have found the relationship between exotic species richness and beta diversity to vary depending on the taxonomic group under study and the spatial scale (McKinney 2004). Among the handful of legacy studies documenting biotic homogenization over time, only one found a significant linkage between exotic gains and biotic homogenization (Rooney et al. 2004). Another found the two uncorrelated, despite substantial gains in exotics at the plot level (Rogers et al. 2008). Clearly, knowledge gaps remain concerning how exotic species influence beta diversity over long time-scales and how their potential effect may vary with changing disturbance levels and/or the characteristics of the ecological community in question.

Increasing human dominance of the landscape results in multiple potential drivers of change in ecological communities, including climate change, eutrophication and habitat fragmentation (Jackson \& Sax 2010). The challenge is to understand which of these drivers are most important in determining which species are 'winners' and 'losers' in a particular region, thus mediating changes in alpha and beta diversity (Olden \& Poff 2003). One way to do this is to use traits of species. For example, if eutrophication via nitrogen deposition is an important driver of change, species associated with high-nutrient conditions should be more likely than others to increase in frequency and/or abundance over time (e.g. Smart et al. 2005).

We investigated changes in local plant community diversity over a period of four decades in a highly developed area of southeastern Vancouver Island, British Columbia, Canada (Fig. 1). In Canada, the highest levels of human population density and biological diversity coincide in the south (Kerr \& Cihlar 2004), and therefore, many areas of southern Canada are priorities for conservation. We resurveyed 184 vegetation plots originally surveyed in the late 1960s. At that time, exotic species were already present in $63 \%$ of plots. Since the first survey, the human population in the region has doubled (Statistics Canada 1969, 2011), the landscape has become increasingly fragmented and new invasive exotic species have emerged. Therefore, we predicted (i) increases in exotic species richness and abundance, declines in native species and a decline in alpha diversity since 1968; (ii) biotic homogenization, driven by the expansion of exotic species across the landscape; and (iii) a shift in community composition favouring disturbance-tolerant species, primarily exotic species. Contrary to our expectations, we found that plot level and total diversity have both increased over forty years, with increases in both exotic and native species, although beta diversity has declined. Despite the spread of exotic species

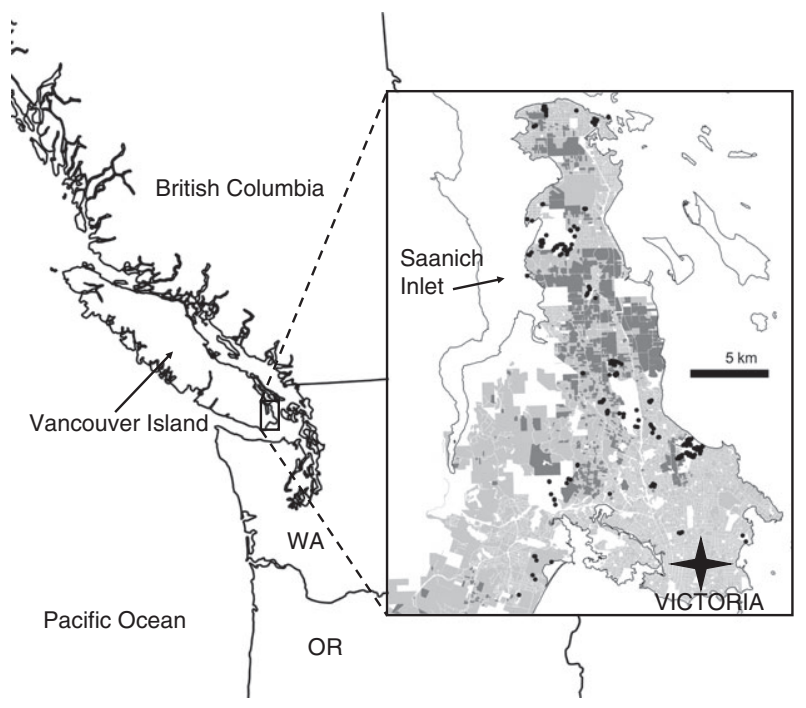

Fig. 1. The study area. Relevé plot locations are indicated by black dots. Light grey shading indicates residential, commercial, industrial or institutional land use. Dark grey shading indicates agricultural land use. Unshaded regions of the peninsula are parks or other green spaces, military land or First Nations reserve land.

across the landscape, we did not find a correlation between exotic invasion and biotic homogenization, finding instead that colonization by native species was a stronger predictor of losses in beta diversity.

\section{Materials and methods}

\section{STUDY AREA}

The Saanich Peninsula is an area of $c .330 \mathrm{~km}^{2}$ including the city of Victoria and partially separated from the bulk of southeastern Vancouver Island by Saanich Inlet (Fig. 1). It became one of the first areas in British Columbia to be settled by Europeans when Victoria was established in 1843. Since then, steady growth in agriculture, logging and suburban development has resulted in a highly fragmented landscape. At the time of the first survey, about one-third of the peninsula outside the city of Victoria still supported forest or savanna vegetation (Roemer 1972). Much less than that remains today (Fig. 1).

Most of the study area is underlain by granitic or volcanic bedrock and covered with a layer of unconsolidated glacial till (Day, Farstad \& Laird 1959). Soils are mainly brunisols and gleysols, which have developed on morainal or marine deposits (Jungen 1985). The Saanich Peninsula is located in the rainshadow of both the Olympic Mountains and the Vancouver Island Mountains, resulting in an unusually warm and dry climate compared with the rest of coastal British Columbia. The climate is described as 'cool Mediterranean', with warm, dry summers and mild, wet winters. Total annual precipitation averages about $87 \mathrm{~cm}$, with only about $10 \mathrm{~cm}$ of this falling in the summer months (June to September).

The Saanich Peninsula lies within the coastal Douglas-fir (CDF) biogeoclimatic zone (Meidinger \& Pojar 1991). Douglas-fir forests are the dominant vegetation characterized by a canopy of Douglas-fir (Pseudotsuga menziesii (Mirb.) Franco), sometimes mixed with Western red cedar (Thuja plicata Donn ex D. Don) or grand fir (Abies grandis (Douglas ex D. Don) Lindl.), and an understorey often 
dominated by salal (Gaultheria shallon Pursh), Oregon-grape (Mahonia aquifolium (Pursh) Nutt. or M. nervosa (Pursh) Nutt.) and sword fern (Polystichum munitum (Kaulf.) C. Presl). In the driest forests, Pacific madrone (Arbutus menziesii Pursh) grows interspersed with Douglas-fir (Flynn 1999). Also, within the CDF zone are oak woodlands and savannas characterized by open grassland and/or shrubs and a sparse canopy of Garry oak trees (Quercus garryana Douglas ex Hook.). Garry oak communities are home to many species that are imperilled provincially or nationally (Fuchs 2001; Parks Canada Agency 2006). Both Douglas-fir forests and Garry oak communities have been reduced in area by more than $90 \%$ since European settlement (Flynn 1999; Lea 2006). Due to the fact that savannas were usually the first areas to be converted to agriculture, current oak community vegetation is now largely confined to higher elevation, steeper areas with shallow soils (Vellend, Bjorkman \& McConchie 2008). The remaining forests of southeastern Vancouver Island also have a much higher density of trees than at the time of European settlement, a consequence of more than a century of fire suppression (Bjorkman \& Vellend 2010).

\section{RESURVEY METHODS}

The original survey was carried out in 1968-1969 (hereafter 1968; Roemer 1972). The abundance of all vascular and non-vascular plant species in 409 relevé plots of $400 \mathrm{~m}^{2}$ area was recorded using the Braun-Blanquet cover-abundance scale (Roemer 1972). Plot locations were subjectively chosen based on homogeneity of vegetation and avoiding highly disturbed areas and young second-growth forests. However, given the widespread logging that occurred on the peninsula prior to the original survey, some relatively early successional areas were sampled by necessity. Plots were not permanently marked; however, a description of their location, slope and aspect and a grid reference on an aerial photo (1:10 000 scale) were recorded for each plot.

In 2009, we relocated and resurveyed 184 of the original relevés. After the original survey, each relevé was classified into one of 28 plant communities in seven associations (Roemer 1972). We limited our resurvey efforts to four associations that cover the portion of the moisture gradient that includes the dominant vegetation type and those of greatest conservation concern: two Garry oak-dominated associations (which we combined and refer to as the 'Garry oak' vegetation type), the Douglas-fir-dominated association ('Douglas-fir' vegetation type) and the Arbutus-Pseudotsuga association ('Arbutus' vegetation type), which represents the transition between Garry oak and Douglas-fir (Roemer 1972).

All relevés were resurveyed between May and August, 2009. Relevés range in elevation from sea level to $c .325 \mathrm{~m}$ above sea level. We resurveyed only where sites were still vegetated and where permission could be obtained from municipal or provincial park authorities or private landowners. The aerial photo grid reference system in conjunction with GPS and advice from the original surveyor $(\mathrm{H}$. L. Roemer, pers. comm.) allowed us to navigate to the approximate location of the plot. We then used the original notes on the slope, aspect, proximity to roads and/or other landscape features to select the most likely site of the original plot. As such, our plots cover the same range of environmental conditions (slope, aspect, elevation, soil type, etc.) as found in the initial survey. We also maximized stand homogeneity within the relocated plot to avoid the potential problem of increased heterogeneity in plots initially established with a preference for homogenous vegetation, given spatial variation in disturbance (Palmer 1993; Ross et al. 2010). We recorded the abundance of all vascular and non-vascular plant species to the nearest one percentage cover, with species covering less than one percentage denoted as 0.5 and very rare species covering much less than one percentage (i.e. only one or two small individuals) denoted as 0.1 .

\section{STATISTICAL ANALYSES}

All cover values for the 1968 and 2009 data were transformed to the percentage cover midpoints of the Braun-Blanquet cover scale, except for species of abundances 0.5 and 0.1 , which were maintained as the lowest two classes of the scale. We standardized plant nomenclature and combined taxa at the level of genus where the accuracy of species identifications was in doubt. Plant nomenclature follows Douglas et al. (1998). We separated vascular plant species into tree species and understorey species, which include grasses, forbs, ferns and shrubs. Understorey species collectively comprise $c .80 \%$ of the full set of species observed, and we focus our analysis on this subset of species.

We used linear mixed-effects models to test the effect of year, vegetation type (as classified in 1968) and their interaction on plot-level understorey species richness. We then used paired $t$-tests to test for a significant change in total species richness, native species richness and exotic species richness between years.

To test the hypothesis of a shift in community composition over time, we used permutational manova (PERMAnova; Anderson 2001; Anderson, Gorley \& Clarke 2008) with year of survey and vegetation type as fixed variables and plot identity as a random variable. We tested for changes in beta diversity between the 2 years across all plots, and within each vegetation type separately, using a distancebased test for homogeneity of multivariate dispersions (PERMDISP; Anderson, Ellingsen \& McArdle 2006). To visualize any shifts in composition or changes in dispersion, we used non-metric multidimensional scaling (NMDS) to ordinate all plots in both years. We used the Bray-Curtis dissimilarity measure because it takes species abundances into account and is recommended for tests of biotic homogenization (McCune \& Grace 2002; Olden \& Rooney 2006).

To test for predictors of changes in beta diversity, we first computed the ecological distance from each plot in species space to the centroid of all plots in both years $(n=368)$. We then calculated the change in this distance by subtracting the value for 2009 from the value for 1968 for each plot. Positive values indicate that a plot shifted towards the average in terms of plant community composition, thereby contributing to biotic homogenization. We then used linear regression to test whether the net change in exotic species per plot between years or the net change in native species per plot between years were correlated with the degree of movement towards the centroid. We also tested for any correlation between the change in number of exotic species and the change in number of native species using Spearman's rank correlation test.

To explore possible drivers of temporal community change, we gathered information on plant traits from various published and unpublished sources. We define 'traits' as both qualitative and quantitative characteristics of plant species and/or their preferred habitats. We chose traits based on hypothesized potential drivers of change in plant communities in the region, including disturbance, fragmentation, climatic warming, increased nitrogen deposition and plant community succession. The traits include disturbance tolerance, origin (exotic or native), soil nutrient regime preference, broad geographic range (ranging primarily to the south of the study area versus study area central in the species' range), seed weight, seed dispersal mechanism, palatability to deer, shade tolerance, form (herbaceous versus shrub) and 
specific leaf area (SLA). For details, see Appendix S1 and Table S1 in Supporting Information.

To determine which species were significantly more frequent and/ or abundant in each of the 2 years, we used an indicator species analysis (Dufrêne \& Legendre 1997) with 1000 randomizations to calculate $P$-values. Significant indicators of 1968 were deemed 'losers', significant indicators of 2009 were deemed 'winners', and species that were not significant indicators of either year were deemed 'no change' species $(P<0.05)$. We then tested for associations between 'winner', 'no change' or 'loser' status and each individual trait value using cumulative logit models. These models preserve the information contained in the ordered nature of the categorical response variable without assuming the distance between categories to be constant (Agresti 2002). To assess whether a particular trait was significantly associated with the indicator status of species, we compared a cumulative logit model with the trait value as an independent variable to a model with intercept only. We limited our analyses to species which occurred in at least 18 plot-year combinations (5\%) and considered a trait to be related to plant indicator status when the difference in AIC (AIC intercept only minus $\mathrm{AIC}_{\text {including trait }}$ ) was greater than 5, which corresponds to strength of evidence at least 12 times stronger than the alternative model (Anderson 2008; Burnham, Anderson \& Huyvaert 2011). We did not attempt to build a model using all traits because the subset of species for which all trait data was available was very small (45 species) and this limited our ability to detect any differences. We also carried out indicator species analysis on each of the three vegetation types separately, to see whether the same species were 'winners' in different vegetation types.

In addition to testing the association of indicator status with individual traits, we calculated the abundance-weighted average trait value for each plot in each year and then used paired tests to determine whether there have been significant shifts over time. The data
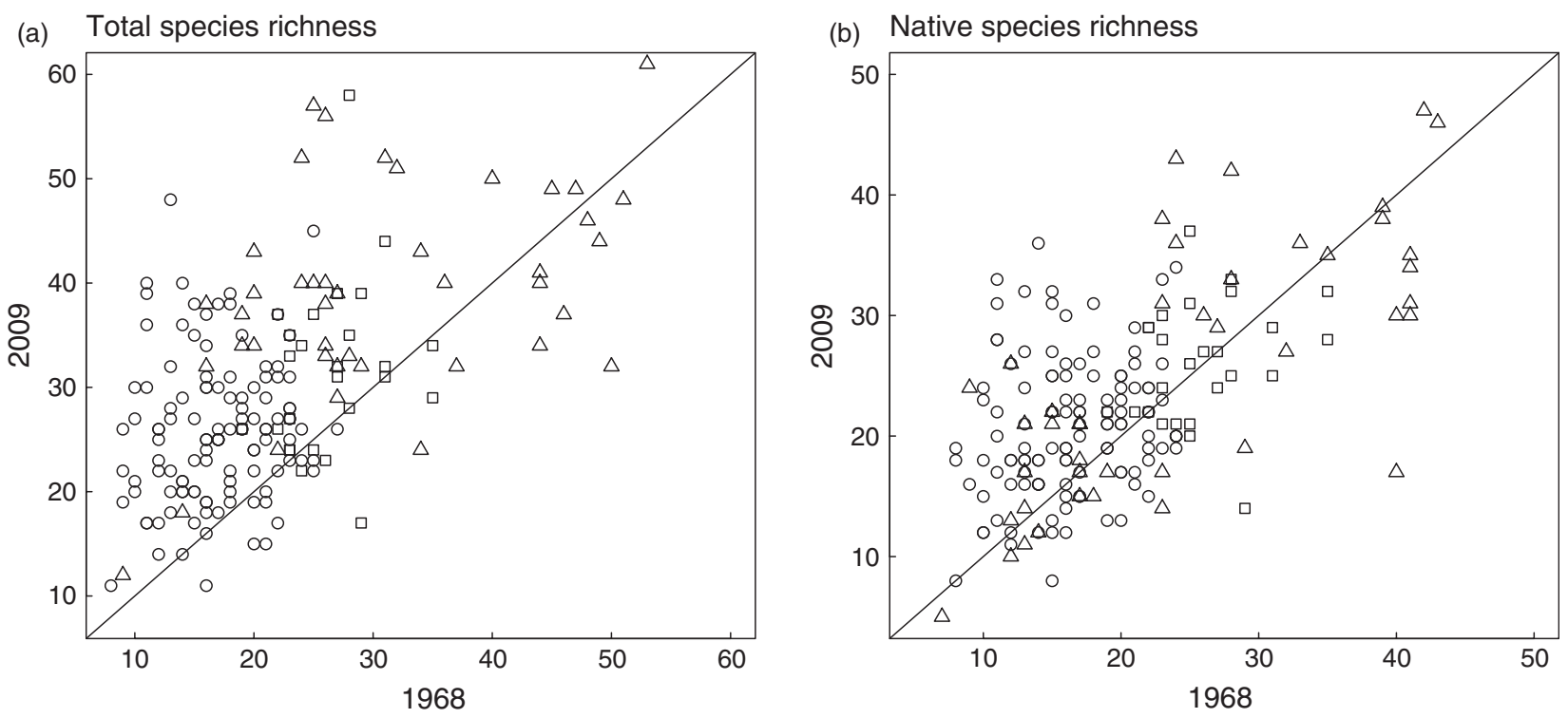

(c) Exotic species richness

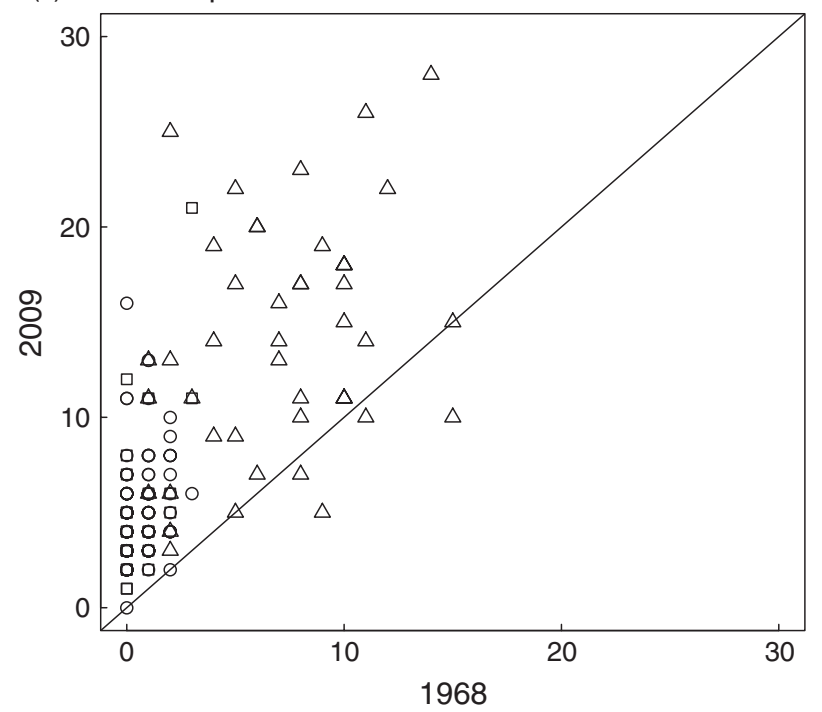

(d) Gains and losses by origin

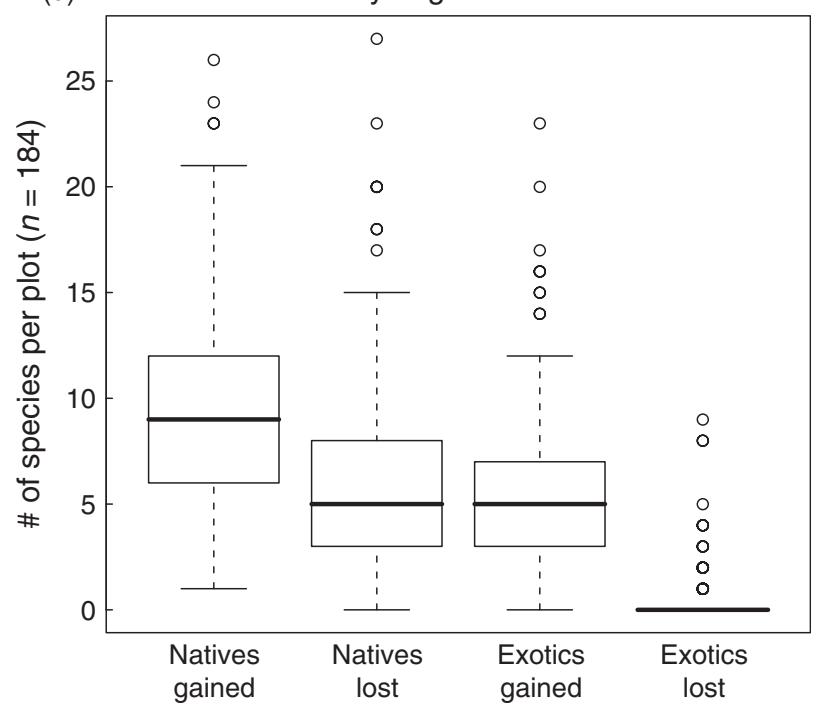

Fig. 2. Plots of (a) total understorey species richness, (b) total native understorey species richness and (c) total exotic understorey species richness in 2009 versus 1968. Circles $=$ Douglas-fir, squares $=$ Arbutus, triangles = Garry oak. The line is the $1: 1$ line; (d) shows the number of species gained and lost per plot by origin. 
often did not meet assumptions of normality, so we used the information-theoretic equivalent to the paired $t$-test (Burnham, Anderson \& Huyvaert 2011) and deemed there to have been a shift in plot-level trait value where the difference in AIC was $>5$. We limited our comparisons to plots in which at least $80 \%$ of the total cover in both years was represented by species for which trait values were available.

Permutational MANOVA and tests for homogeneity of multivariate dispersions were carried out using PRIMER version 6 with PERMANO$\mathrm{VA}^{+}$(Clarke \& Gorley 2006). All other analyses were carried out in $\mathrm{R}$, using the 'vegan' package for NMDS ordination, the 'labdsv' package for indicator species analysis and the 'ordinal' package for cumulative logit models (Oksanen et al. 2009; Roberts 2010; R Core Development Team 2012; Christensen 2013).

\section{Results}

The total number of understorey species recorded increased from 179 in 1968 to 237 in 2009. Eighty-seven species were new to this set of plots, while 29 of the original species were not recorded in 2009. The total number of native species increased from 146 to 149 , and the total number of exotic species increased from 33 to 82 . With the exception of a few very recently naturalized exotic species, all the species new to our sample of plots were present somewhere on the Saanicheninsula in 1968 (H. L. Roemer, pers. comm.).

The best linear mixed-effects model for species richness included year, vegetation type and their interaction. Understorey species richness increased by an average of eight species, from 21 to 29 species on average per plot (paired $t=12.5946$, d.f. $=183, P<2.2 \mathrm{e}-16$; Fig. 2a). Native species also increased, from an average of 19 to 22 native species per plot (paired $t=6.0091$, d.f. $=183, P=4.935 \mathrm{e}-09$; Fig. 2b), and exotic species increased from an average of 2 to 7 exotic species per plot (paired $t=17.2808$, d.f. $=183, P=2.2 \mathrm{c}-16$; Fig. 2c). On average, each plot gained 15 species (9 native and 6 exotic) and lost seven species (just over 6 native and less than one exotic; Fig. 2d).

There was a shift in plant community composition and a decline in beta diversity between 1968 and 2009 (Fig. 3). PERMANOVA confirmed that plots in different years and different habitats are significantly different in terms of understorey plant species composition (Table 1). PERMDISP showed a significant decline in heterogeneity between 1968 and 2009 for all plots $\left[F_{1,366}=9.4394, P=0.006\right.$, mean Bray-Curtis

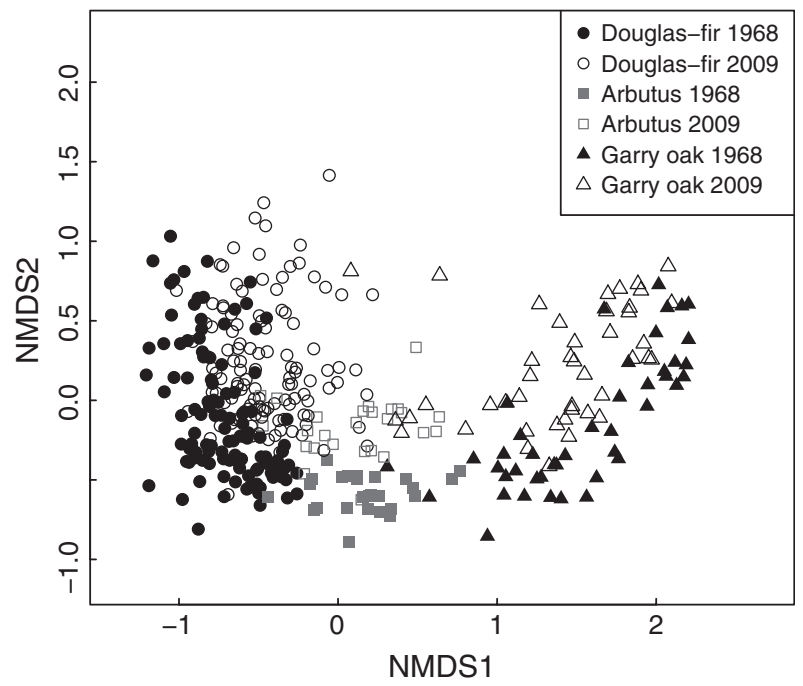

Fig. 3. Non-metric multidimensional scaling ordination of all plots in 1968 and 2009 in understorey species space. The Bray-Curtis dissimilarity measure was used with double Wisconsin standardization and square root transformation. To avoid a maximum dissimilarity threshold, flexible shortest paths were found between plots with no species in common (Oksanen et al. 2009). The number of dimensions was set to 3 . The ordination has been centred, the axes scaled to half-change units and the configuration rotated so that the variation among plots is maximized on axis NMDS1. Stress in three dimensions is 12.37.

distance to centroid $(1968)=59.744$, mean Bray-Curtis distance to centroid $(2009)=56.763$ ]. However, when analysing vegetation types separately, only Douglas-fir-dominated plots showed a significant reduction in dispersion over time, while Arbutus plots showed a non-significant increase in multivariate dispersion, and Garry oak plots showed a non-significant decline in multivariate dispersion (Table 2). The average pairwise Bray-Curtis similarity between vegetation types increased from 12.54 in 1968 to 15.10 in 2009. Seventy-one percentage of plots moved towards the overall centroid over time. The compositional shift of each plot towards the average was uncorrelated with the change in number of exotic species per plot $(F=2.303, \quad P=0.1309, \quad$ adjusted $R^{2}=0.007$; Fig. 4a), but positively correlated with the change in number of native species per plot $(F=12.53$, $P<0.001$, adjusted $R^{2}=0.05929$; Fig. 4b). The change in number of native species was positively correlated with the change in exotic species (Spearman's rank correlation coefficient: $\rho=0.30977, P<0.0001)$.

Table 1. Results of a PERmanova (Anderson 2001) with year of survey and vegetation type as fixed variables and plot identity as a random variable. The analysis was performed using Bray-Curtis dissimilarities on untransformed, non-relativized abundance data, with sequential sums of squares and 999 permutations

\begin{tabular}{lrrrrrr}
\hline Factor & Degrees of freedom & \multicolumn{1}{l}{ SS } & \multicolumn{1}{c}{ MS } & Pseudo-F & $P($ perm $)$ & No of unique permutations \\
\hline Year & 1 & 27070 & 27070 & 15.4 & 0.001 & 999 \\
Vegetation type & 2 & 221210 & 110600 & 27.6 & 0.001 & 998 \\
Plot identity & 181 & 725660 & 4009.2 & 2.3 & 0.001 & 994 \\
Year x vegetation type & 2 & 17223 & 8611.3 & 4.9 & 0.001 & 998 \\
Residual & 181 & 317250 & 1752.7 & & & \\
Total & 367 & 1308400 & & & & \\
\hline
\end{tabular}


Table 2. Results of pairwise comparisons between plots of the same vegetation type in different years in terms of multivariate dispersions using PERMDISP and the Bray-Curtis similarity measure. $P$-values were calculated based on 999 permutations

\begin{tabular}{lllll}
\hline & $\begin{array}{l}\text { Mean } \\
\text { distance } \\
\text { to centroid } \\
(1968)\end{array}$ & $\begin{array}{l}\text { Mean } \\
\text { distance } \\
\text { to centroid } \\
(2009)\end{array}$ & $t$ & $P$ \\
Vegetation type & 54.5 & 50.7 & 3.19 & $0.007^{*}$ \\
\hline Douglas-fir $(n=115)$ & 40.6 & 44.2 & 1.15 & 0.323 \\
Arbutus $(n=27)$ & 58.1 & 55.3 & 1.89 & 0.072 \\
Garry oak $(n=43)$ & & & & \\
\hline
\end{tabular}
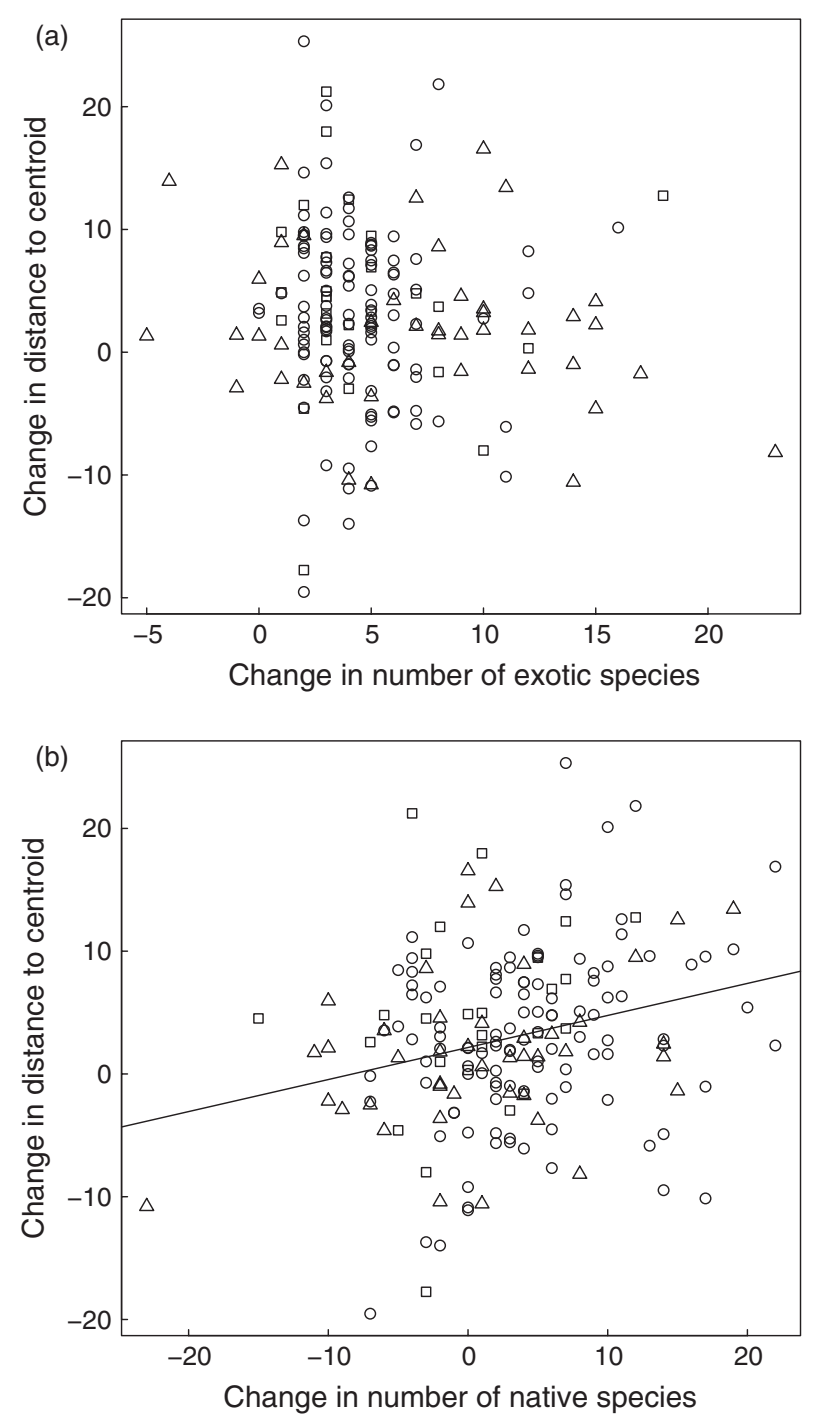

Fig. 4. The relationship between the change in distance from the overall multivariate species centroid over time and (a) change in number of exotic species present and (b) change in number of native species present from 1968 to 2009. The line in (b) is the best fit line by linear regression; there was no significant correlation in (a). Positive change in the distance to centroid indicates a shift towards increased similarity with other plots. Circles $=$ Douglas-fir plots, squares $=$ Arbutus plots, triangles $=$ Garry oak plots.
There were 101 understorey species with a frequency of at least 5\% across all plot-year combinations. Twenty-nine species were identified as overall 'winners', 63 showed no significant change in frequency and/or abundance, and nine were deemed 'losers' (see Table S2). Cumulative logit models showed that 'winners' were more likely to be exotic, disturbance tolerant, shade tolerant and shrubs (Fig. 5; Table 3). The paired tests for change in abundance-weighted trait values between survey periods also found significant increases in abundance-weighted exoticness and disturbance tolerance, but no significant change in abundance-weighted shade tolerance or shrub cover over time (Table 3).

\section{Discussion}

Our study shows a striking increase in taxonomic diversity of plant communities on the Saanich Peninsula over the last four decades, with gamma diversity increasing by $32 \%$ and alpha diversity increasing by $38 \%$. However, these increases were accompanied by a significant decline in beta diversity over time. This is the first North American study we know of to find this pattern, although it has been observed in Europe (Van Calster et al. 2007).

The total number of exotic species more than doubled, and each plot gained six exotic species on average, but these dramatic gains do not seem to be the cause of biotic homogenization. In fact, the shift of plots towards a more similar plant community composition was correlated with gaining native species (although the proportion of variation explained was low: Fig. 4b). The tendency of a colonizing species to cause biotic homogenization depends on the frequency of occurrence of the species elsewhere. If a plot gains a species that is found in very few other plots, this can lead to biotic differentiation (Olden \& Poff 2003; McKinney 2004). For this reason, the role of exotic species in homogenization has usually been found to be greater at larger scales, where native floras of distant territories have very few species in common and thus are made more similar by the addition of a few widespread exotic species (McKinney 2008). Of the 101 species included in our indicator species analysis, native species were found in an average of 45 plots in 1968, while exotic species had an average frequency of only 14 plots. Therefore, a gain in a native species was more likely to cause homogenization. The importance of gains in native species is supported by an examination of the indicator species of Douglas-fir plots alone, which was the only vegetation type to show biotic homogenization when analysed alone. The Douglas-fir vegetation type was the only type that showed an increase in total number of native species across all plots (from 69 to 94), and the majority of the 'winners' in Douglas-fir plots are native (Table S2). Local gains in native species have been shown to drive biotic homogenization where exotics are rare or absent (e.g. Van Calster et al. 2007; Naaf \& Wulf 2010), however, even with substantial gains in exotics at the local and regional scale, it appears that the spread of native species on the Saanich Peninsula has promoted the reduction in beta diversity. 

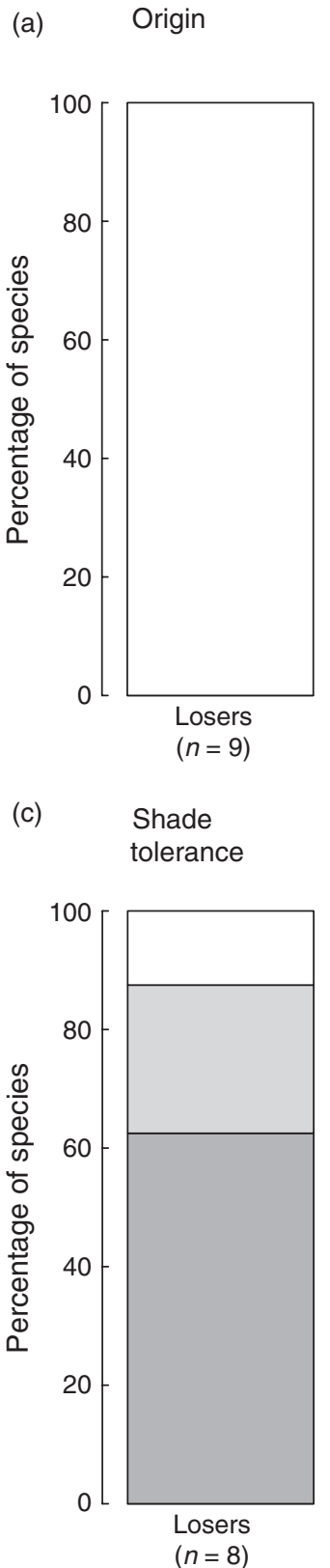

$\square$ Exotic $\square$ Native

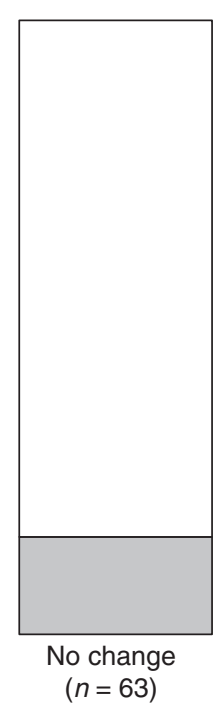

Tolerant Tol.-intol.

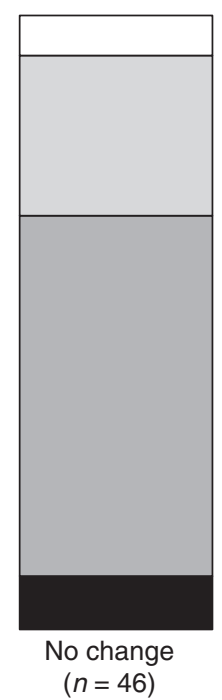

(b)

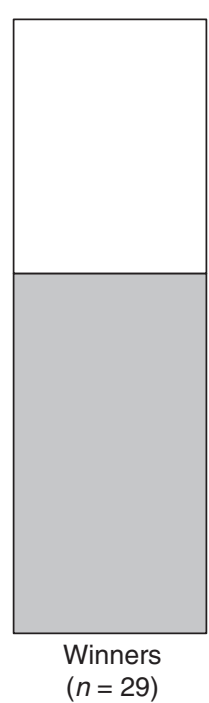

$\square$ Intolerant $\square$ Very intolerant

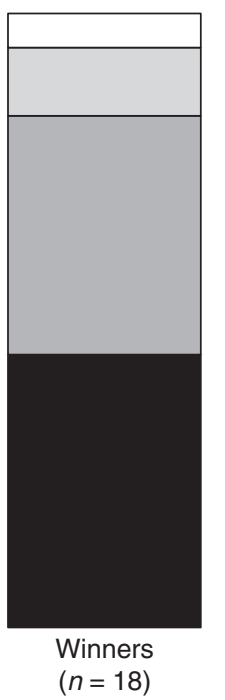

(d)

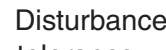

tolerance
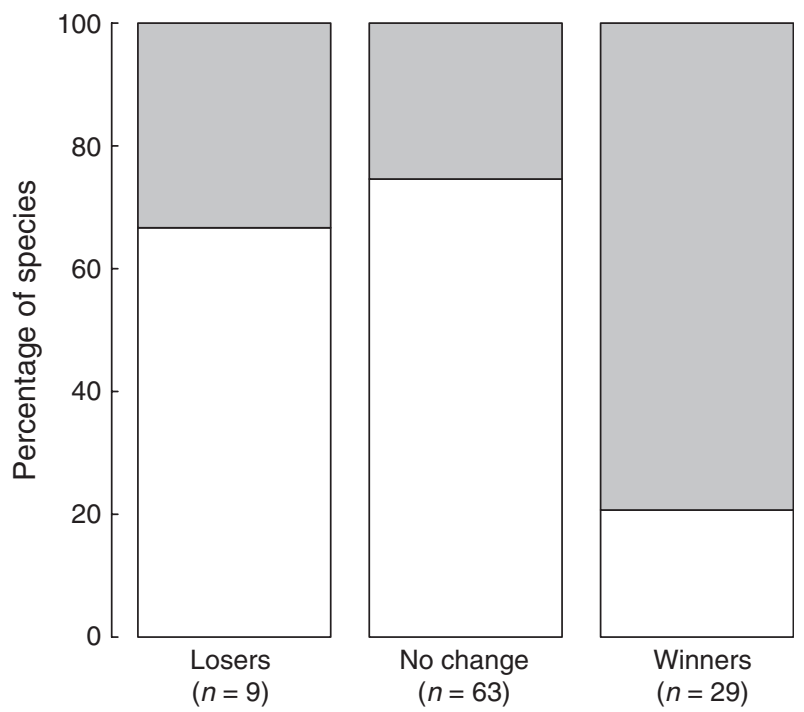

Growth form
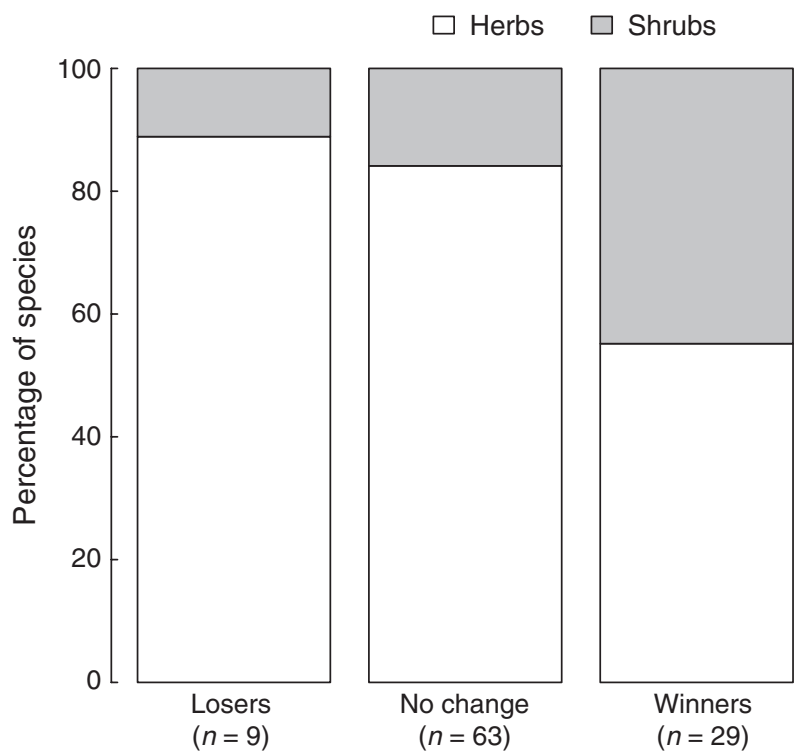

Fig. 5. The percentage of species in each category for the four traits that were found to be strongly associated with indicator status: (a) origin, (b) disturbance tolerance, (c) shade tolerance, and (d) growth form. The number of species for which trait values were available is listed below each bar (total $n=101)$.

The overall 'winners' were more likely to be exotic, disturbance tolerant, shade tolerant and shrubs. For example, the most successful exotic species include Ilex aquifolium L., Hedera helix L., and Daphne laureola L., all shade-tolerant shrubs, each of which has colonized more than 70 plots since the first survey (see Table S2). Shade-tolerant exotic 'winners' also include the intriguing orchid Epipactis helleborine (L.) Crantz, which was not found in any plot in 1968 but has since colonized 80 plots (Table S2). Cytisus scoparius (L.) Link has been an overwhelming 'winner' in Garry oak vegetation where conditions are less shaded.

Native 'winners' include the disturbance-tolerant shrubs Rubus ursinus subsp. macropetalus Cham. \& Schltdl. (Doug- las ex Hook.) Roy L. Taylor \& MacBryde, Oemleria cerasiformis (Torr. \& A. Gray ex Hook. \& Arn.) Landon and Amelanchier alnifolia (Nutt.) Nutt. ex M. Roem., and herbs Nemophila parviflora Douglas ex. Benth. and Galium triflorum Michx. 'Losers' include herbs that are not disturbance tolerant, such as Trillium ovatum Pursh, Erythronium oreganum Applegate and Tiarella trifoliata L., and the shade-intolerant species Carex inops L.H. Bailey and Bromus carinatus Hook. \& Arn.

These results suggest that direct human disturbance has been the most important driver of plant community change in this region in the past 40 years, with some role for increased shading and infilling by shrubs. We did not find evidence 
Table 3. Results of cumulative logit model comparisons for the association between indicator status (loser, no change, winner) and traits, and for paired tests of the change in abundance-weighted average trait value per plot

\begin{tabular}{|c|c|c|c|c|c|c|}
\hline \multirow[b]{2}{*}{ Trait } & \multicolumn{3}{|c|}{$\begin{array}{l}\text { Association with indicator value } \\
\text { (cumulative logit model) }\end{array}$} & \multicolumn{3}{|c|}{$\begin{array}{l}\text { Change in abundance-weighted average } \\
\text { plot value (paired tests) }\end{array}$} \\
\hline & $\begin{array}{l}\text { Explained deviance } \\
\left.\text { (pseudo } R^{2}\right)(\%)\end{array}$ & $\begin{array}{l}\Delta \text { AIC (intercept } \\
\text { alone versus model } \\
\text { with trait) }{ }^{\dagger}\end{array}$ & $\begin{array}{l}n \text { (No } \\
\text { of spp.) }\end{array}$ & $\begin{array}{l}\text { Mean of the paired } \\
\text { differences } \\
(2009-1968)\end{array}$ & $\triangle \mathrm{AICc}$ & $\begin{array}{l}n \text { (No } \\
\text { of plots) }\end{array}$ \\
\hline Origin $($ exotic $=0$, native $=1)$ & 12.54 & $19.99 *$ & 101 & -0.097 & $36.05^{*}$ & 184 \\
\hline $\begin{array}{l}\text { Disturbance tolerance } \\
(\text { not tolerant }=0, \text { tolerant }=1)\end{array}$ & 11.27 & $17.76^{*}$ & 101 & 0.074 & $14.27 *$ & 184 \\
\hline Form $(0=$ herb, $1=$ shrub $)$ & 5.28 & $7.26 *$ & 101 & 0.035 & 0.77 & 184 \\
\hline $\begin{array}{l}\text { Shade tolerance } \\
(1=\text { very tolerant } \rightarrow 5=\text { very intolerant })\end{array}$ & 5.61 & $5.08 *$ & 72 & -0.036 & 2.94 & 152 \\
\hline Seed weight (g per 1000 seeds) & 3.23 & 2.58 & 83 & 1.295 & 2.54 & 86 \\
\hline Specific leaf area (SLA) $\left(\mathrm{mm}^{2} / \mathrm{g}\right)$ & 0.80 & -0.88 & 86 & 0.571 & -1.43 & 177 \\
\hline Range $(1=$ southerly, $2=$ central $)$ & 0.07 & -1.87 & 101 & 0.036 & 0.70 & 184 \\
\hline $\begin{array}{l}\text { Seed dispersal mechanism } \\
(0=\text { none, } 1=\text { wind, } 2=\text { vertebrate })\end{array}$ & 0.30 & -1.79 & 37 & 0.022 & -1.83 & 34 \\
\hline $\begin{array}{l}\text { Deer palatability } \\
(0=\text { not eaten } \rightarrow 3=\text { highly palatable })\end{array}$ & 3.46 & 0.93 & 50 & 0.034 & -1.26 & 101 \\
\hline $\begin{array}{l}\text { Nutrient regime } \\
(1=\text { oligotrophic } \rightarrow 6=\text { hypereutrophic })\end{array}$ & 0.11 & -1.85 & 82 & 0.042 & 1.75 & 150 \\
\hline
\end{tabular}

*Strong evidence $(\triangle \mathrm{AIC}>5)$ that a trait is associated with indicator species status or that abundance-weighted mean plot-level values for that trait have shifted from 1968 to 2009.

$\dagger$ Negative values indicate that the model with intercept only had a lower AIC than the model with the trait included.

that plant communities are shifting in terms of deer palatability, geographic range characteristics, soil nutrient regime preferences, seed dispersal mechanisms or specific leaf area. However, we realize that our ability to detect shifts in these traits could be limited when more than one mechanism is operating that favour different extremes of a trait. For example, our winners include many heavy-seeded bird-dispersed shrubs, such as A. alnifolia, I. aquifolium and O. cerasiformis, which may be favoured with forest fragmentation. However, tiny-seeded species associated with mature forests, such as Monotropa uniflora L., and Corallorhiza maculata (Raf.) Raf. are also on the winning list, probably as a result of forest maturation over the past four decades, in particular, in plots that were at an earlier seral stage when first surveyed. We also recognize that other unmeasured characteristics correlated with our measured traits may influence the relative success of a species over the 4-decade period.

Our finding that 'winners' were more likely to be shade tolerant suggests a potential role for succession in driving the changes we observed in plant community composition. However, the majority of our plots were already considered mature in 1968. In addition, the enormous increase in understorey richness in Douglas-fir plots in particular (Fig. 2), the significant tendency for disturbance-tolerant species to be winners (Fig. 5b) and the significant shift in abundance-weighted plotlevel disturbance tolerance are trends opposite to what would be expected if succession was the dominant driver. The tendency for 'winners' to be shade tolerant was also not strong enough to produce a significant increase in plot-level abundance-weighted shade tolerance (Table 3). Thus, although succession may be playing a role in some cases, our results implicate disturbance as the dominant driver of change.
Exotic species were much more likely than natives to be 'winners': $58.6 \%$ of exotic species were 'winners', while only $16.2 \%$ of native species were. This may be explained if relatively recently introduced exotics had simply not yet filled in their potential ranges in 1968 (an 'invasion debt'; Bennett et al. 2013). We also note that the trait 'exotic' is highly correlated with disturbance tolerance. In our entire data set, $93 \%$ of the exotic species are disturbance tolerant, but only $23 \%$ of native species are disturbance tolerant. Although many exotic species have been highly successful over the time frame of our survey, they are not yet the driving force behind biotic homogenization, nor do they appear responsible for extirpations of native species, given the positive correlation of temporal change between exotic and native species richness. Our indicator species analyses identified many more 'winners' than 'losers'. The ratio of extirpated to colonizing species (the 'extinction pressure', sensu Olden \& Poff 2003) is low for our data set ( 0.33 for all plots pooled, 0.46 on a plot-level basis), indicating that at this time, colonizations are not causing high levels of extinction. Of course, this could change in the future, especially if there are time lags between colonization and resulting extinctions, resulting in an extinction debt (Vellend et al. 2006; Jackson \& Sax 2010).

Finally, we would like to emphasize the importance of the breadth of our environmental gradient to our finding of biotic homogenization. Had we considered only Garry oak plots, for example, we would have found a shift in plant species composition, but no significant biotic homogenization. The changes occurring on this landscape are resulting in a blurring of the distinction between plant community types. The total length of the dominant vegetation gradient has declined from 
3.41 to 3.11 half-change units (Fig. 3), and the average BrayCurtis similarity between vegetation types has increased. This is a trend that has also recently been observed over 50 years in upland plant communities in Scotland (Ross et al. 2012). The reason why the increase in alpha diversity led to significant biotic homogenization among Douglas-fir plots but not the other vegetation types may be due to different starting points on the gradient from less to more disturbed (e.g. Smart et al. 2006), a result of differences in land use history. Most Garry oak plots were already highly invaded by exotics and isolated in small fragments at the time of the first survey, whereas Douglas-fir plots were still found in more intact, contiguous forest areas where exotic species and disturbanceassociated natives were extremely rare. As a result, Douglas-fir plots saw a much more dramatic relative increase in species and significant biotic homogenization with respect to each other. The ability to detect biotic homogenization not only depends on the spatial and temporal scale at which it is tested (e.g. Olden \& Poff 2003; Baiser et al. 2012), but also the length of the plant community gradient over which it is examined.

\section{Synthesis}

A long-term perspective is crucial to understand how increasing human dominance is changing plant communities worldwide. The majority of long-term studies to date have shown a decline in plant diversity over time. Our study shows that human-mediated disturbance is an important driver of plant community changes in recent decades on the Saanich Peninsula, but the net result has actually been an increase in richness for both natives and exotics. However, these increases were accompanied by a decline in beta diversity across the landscape promoted by the colonization of common, disturbance-tolerant native species. This biotic homogenization is equivalent to a shrinking of the vegetation gradient and may not have been detected if the full range of vegetation types had not been included.

\section{Acknowledgements}

We gratefully acknowledge Dr. Hans Roemer for advising us on plot relocation, sharing his datasheets and notes from the original survey, assisting with plant species identifications and offering valuable comments on an earlier draft of this manuscript. We thank the following for permission to access plots: BC Parks, District of Central Saanich, Capital Regional District, District of North Saanich, District of Saanich, City of Victoria, Royal Roads University, Hertzberg Institute of Astrophysics, Royal Oak Burial Park, Victoria International Airport, University of Victoria, the Pauquachin Nation and several private landowners. Anthony Ho and Alice Cang assisted with data collection. Dr. Will Cornwell kindly shared his SLA data base for plants of the region. S. Smart and one anonymous referee provided helpful comments on an earlier draft. This research was funded by the Natural Sciences and Engineering Research Council of Canada (Postgraduate Scholarship to J.L.M., Discovery Grant to M.V.).

\section{References}

Aggemyr, E. \& Cousins, S.A.O. (2012) Landscape structure and land use history influence changes in island plant composition after 100 years. Journal of Biogeography, 39, 1645-1656.
Agresti, A. (2002) Categorical Data Analysis. Wiley, New York, USA.

Anderson, M.J. (2001) A new method for non-parametric multivariate analysis of variance. Austral Ecology, 26, 32-46.

Anderson, D.R. (2008) Model Based Inference in the Life Sciences: A Primer on Evidence. Springer, New York, USA.

Anderson, M.J., Ellingsen, K.E. \& McArdle, B.H. (2006) Multivariate dispersion as a measure of beta diversity. Ecology Letters, 9, 683-693.

Anderson, M.J., Gorley, R.N. \& Clarke, K.R. (2008) PERMANOVA+ for PRIMER: Guide to Software and Statistical Methods. PRIMER-E, Plymouth, UK.

Baiser, B., Olden, J.D., Record, S., Lockwood, J.L. \& McKinney, M.L. (2012) Pattern and process of biotic homogenization in the New Pangaea. Proceedings of the Royal Society B: Biological Sciences, 279, 4772-4777.

Bennett, J.R., Vellend, M., Lilley, P.L., Cornwell, W.K. \& Arcese, P. (2013) Abundance, rarity and invasion debt among exotic species in a patchy ecosystem. Biological Invasions, 15, 707-716.

Bjorkman, A.D. \& Vellend, M. (2010) Defining historical baselines for conservation: ecological changes since European settlement on Vancouver Island, Canada. Conservation Biology, 24, 1559-1568.

Burnham, K.P., Anderson, D.R. \& Huyvaert, K.P. (2011) AIC model selection and multimodel inference in behavioral ecology: some background, observations, and comparisons. Behavioral Ecology and Sociobiology, 65, 23-35.

Butchart, S.H.M., Walpole, M., Collen, B., van Strien, A., Scharlemann Jr, P.W., Almond, R.E.A. et al. (2010) Global Biodiversity: indicators of Recent Declines. Science, 328, 1164-1168.

Christensen, R.H.B. (2013) Ordinal: regression models for ordinal data. R package version 2012.09-11.

Clarke, K.R. \& Gorley, R.N. (2006) Primer v6 Permanova+. Primer-E Ltd., Plymouth, UK.

Damschen, E.I., Harrison, S. \& Grace, J.B. (2010) Climate change effects on an endemic-rich edaphic flora: resurveying Robert H. Whittaker's Siskiyou sites (Oregon, USA). Ecology, 91, 3609-3619.

Day, J.H., Farstad, L. \& Laird, D.G. (1959) Soil survey of Southeast Vancouver Island and Gulf Islands, British Columbia. Research Branch, Canada Department of Agriculture, Ottawa, ON, Canada.

Douglas, G.W., Straley, G.B., Meidinger, D. \& Pojar, J. (1998) Illustrated Flora of British Columbia. Ministry of Environment, Lands and Parks and Ministry of Forests, Victoria, BC, Canada.

Dufrêne, M. \& Legendre, P. (1997) Species assemblages and indicator species: the need for a flexible asymmetrical approach. Ecological Monographs, 67, 345-366.

Flynn, S. (1999) Ecosystems in British Columbia at Risk: Coastal Douglas-fir Ecosystems. Ministry of Environment, Lands and Parks, BC, Canada.

Fuchs, M.A. (2001) Towards a Recovery Strategy for Garry Oak and Associated Ecosystems in Canada: Ecological Assessment and Literature Review. Environment Canada, Canadian Wildlife Service, Pacific and Yukon Region.

Jackson, S.T. \& Sax, D.F. (2010) Balancing biodiversity in a changing environment: extinction debt, immigration credit and species turnover. Trends in Ecology \& Evolution, 25, 153-160.

Jungen, J.R. (1985) Soils of Southern Vancouver Island. Ministry of Environment, Victoria, BC, Canada.

Keith, S.A., Newton, A.C., Morecroft, M.D., Bealey, C.E. \& Bullock, J.M. (2009) Taxonomic homogenization of woodland plant communities over 70 years. Proceedings of the Royal Society B: Biological Sciences, 276, 3539-3544.

Kerr, J.T. \& Cihlar, J. (2004) Patterns and causes of species endangerment in Canada. Ecological Applications, 14, 743-753.

Lea, T. (2006) Historical Garry oak ecosystems of Vancouver Island, British Columbia, pre-European contact to the present. Davidsonia, 17, 34-50.

McCune, B. \& Grace, B. (2002) Analysis of Ecological Communities. MjM Software Design, Gleneden Beach, OR, USA.

McKinney, M.L. (2004) Do exotics homogenize or differentiate communities? Roles of sampling and exotic species richness. Biological Invasions, 6, 495504.

McKinney, M.L. (2008) Do humans homogenize or differentiate biotas? It depends. Journal of Biogeography, 35, 1960-1961.

McKinney, M.L. \& Lockwood, J.L. (1999) Biotic homogenization: a few winners replacing many losers in the next mass extinction. Trends in Ecology \& Evolution, 14, 450-453.

Meidinger, D. \& Pojar, J. (1991) Ecosystems of British Columbia. BC Ministry of Forests, Victoria, BC, Canada.

Naaf, T. \& Wulf, M. (2010) Habitat specialists and generalists drive homogenization and differentiation of temperate forest plant communities at the regional scale. Biological Conservation, 143, 848-855. 
Oksanen, J., Kindt, R., Legendre, P., O'Hara, B., Simpson, G.L., Solymos, P., Henry, M., Stevens, H. \& Wagner, H. (2009) Vegan: community ecology package. $\mathrm{R}$ package version 1.15-4.

Olden, J.D. \& Poff, N.L. (2003) Toward a mechanistic understanding and prediction of biotic homogenization. The American Naturalist, 162, 442-460.

Olden, J.D. \& Rooney, T.P. (2006) On defining and quantifying biotic homogenization. Global Ecology and Biogeography, 15, 113-120.

Palmer, M.W. (1993) Potential biases in site and species selection for ecological monitoring. Environmental Monitoring and Assessment, 26, 277-282.

Parks Canada Agency (2006) Recovery Strategy for Multi-Species at Risk in Garry Oak Woodlands in Canada. Species at Risk Act Recovery Strategy Series, Parks Canada Agency, Ottawa, ON, Canada.

R Core Development Team (2012) R: A language and environment for statistical computing. R Foundation for Statistical Computing, Vienna, Austria.

Roberts, D.W. (2010) Package 'labdsv': ordination and multivariate analysis for ecology. R package version 1.4-1.

Roemer, H.L. (1972) Forest vegetation and environments on the Saanich peninsula, Vancouver Island. $\mathrm{PhD}$ Thesis, University of Victoria, Victoria, BC, Canada

Rogers, D.A., Rooney, T.P., Olson, D. \& Waller, D.M. (2008) Shifts in southern Wisconsin forest canopy and understory richness, composition, and heterogeneity. Ecology, 89, 2482-2492.

Rooney, T.P., Wiegmann, S.M., Rogers, D.A. \& Waller, D.M. (2004) Biotic impoverishment and homogenization in unfragmented forest understory communities. Conservation Biology, 18, 787-798.

Ross, L.C., Woodin, S.J., Hester, A., Thompson, D.B.A. \& Birks, H.J.B (2010) How important is plot relocation accuracy when interpreting re-visitation studies of vegetation change? Plant Ecology \& Diversity, 3, 1-8.

Ross, L.C., Woodin, S.J., Hester, A.J., Thompson, D.B.A. \& Birks, H.J.B. (2012) Biotic homogenization of upland vegetation: patterns and drivers at multiple spatial scales over five decades. Journal of Vegetation Science, 23 755-770.

Sax, D.F. \& Gaines, S.D. (2003) Species diversity: from global decreases to local increases. Trends in Ecology \& Evolution, 18, 561-566.

Smart, S.M., Bunce, R.G.H., Marrs, R., Le Duc, M., Firbank, L.G., Maskell, L.C., Scott, W.A., Thompson, K. \& Walker, K.J. (2005) Large-scale changes in the abundance of common higher plant species across Britain between 1978, 1990 and 1998 as a consequence of human activity: tests of hypothesised changes in trait representation. Biological Conservation, 124, 355-371.

Smart, S.M., Thompson, K., Marrs, R.H., Le Duc, M.G., Maskell, L.C. \& Firbank, L.G. (2006) Biotic homogenization and changes in species diversity across human-modified ecosystems. Proceedings of the Royal Society B, 273, 2659-2665.

Statistics Canada (Dominion Bureau of Statistics) (1969) Canada Year Book 1969. Queen's Printer, Ottawa, ON, Canada.

Statistics Canada (2011) Canada Year Book 2011. Minister of Industry, Ottawa, ON, Canada.

Van Calster, H., Baeten, L., De Schrijver, A., De Keersmaeker, L., Rogister, J.E., Verheyen, K. \& Hermy, M. (2007) Management driven changes (19672005 ) in soil acidity and the understorey plant community following conversion of a coppic-with-standards forest. Forest Ecology and Management, 241, 258-271.

Vellend, M., Bjorkman, A.D. \& McConchie, A. (2008) Environmentally biased fragmentation of oak savanna habitat on southeastern Vancouver Island, Canada. Biological Conservation, 141, 2576-2584.

Vellend, M., Verheyen, K., Jacquemyn, H., Kolb, A., Van Calster, H., Peterken, G. \& Hermy, M. (2006) Extinction debt of forest plants persists for more than a century following habitat fragmentation. Ecology, 87, 542-548.

Vellend, M., Brown, C.D., Kharouba, H.M., McCune, J.L. \& Myers-Smith, I.H. (2013) Historical ecology: using unconventional data sources to test for impacts of global environmental change. American Journal of Botany, 100, 1294-1305.

Received 11 April 2013; accepted 14 August 2013

Handling Editor: Jason Fridley

\section{Supporting Information}

Additional Supporting Information may be found in the online version of this article:

Appendix S1. Compilation of plant trait data.

Table S1. Hypothesized drivers of plant community change, expected shifts in plant traits, and the percentage of species for which data was available for each trait.

Table S2. Indicator species analysis results. 